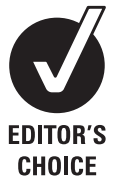

CHOICE

\title{
The Trevo device: preclinical data of a novel stroke thrombectomy device in two different animal models of arterial thrombo-occlusive disease
}

\author{
Raul G Nogueira, ${ }^{1}$ Elad I Levy, ${ }^{2}$ Matthew Gounis, ${ }^{3}$ Adnan H Siddiqui ${ }^{2}$
}

\begin{abstract}
${ }^{1}$ Departments of Neurology, Neurosurgery and Radiology, Marcus Stroke and Neuroscience Center, Grady Memorial Hospital, Emory University School of Medicine, Atlanta, Georgia, USA ${ }^{2}$ Department of Neurosurgery, State University of New York at Buffalo, Buffalo, New York, USA ${ }^{3}$ Department of Radiology, University of Massachusetts, Worcester, Massachusetts, USA
\end{abstract}

\section{Correspondence to} Dr R G Nogueira, Departments of Neurology, Neurosurgery and Radiology, Emory University School of Medicine, Atlanta, GA 30303, USA:

rnoguei@emory.edu

Received 17 April 2011 Revised 11 June 2011 Accepted 9 July 2011 Published Online First 20 August 2011
ABSTRACT

Background The currently available mechanical devices fail to achieve recanalization in as many as $20-40 \%$ of proximal arterial occlusion strokes.

Objective The preclinical evaluation of the safety and efficacy of a novel thrombectomy device designed to achieve immediate flow restoration by quickly removing clot is reported.

Methods Four confirmatory animal studies were performed with the Trevo device (Concentric Medical Inc, Mountain View, California, USA) in the swine $(n=2)$ and canine $(n=1)$ models of arterial thrombo-occlusive disease employing autologous thrombin generated thrombi. The angiographic response and the degree of device-clot incorporation were evaluated. High resolution flat panel three-dimensional CT was performed to further define the in vivo device-thrombus-vessel interaction. Finally, samples of three swine vessels treated with six passes of the device were explanted for histopathological analysis.

Results A total of 16 clots of variable hardness and consistency were implanted in a variety of vascular settings, including the swine internal maxillary, lingual and forelimb arteries as well as the canine external carotid and vertebral arteries. Thrombolysis in Myocardial Infarction (TIMI) 2-3 reperfusion was achieved in all cases immediately after device deployment. All 16 clots were retrieved after one $(n=15)$ or two $(n=1)$ passes with the device. Histopathological analysis demonstrated severe disruption of the intima but no hemorrhage of media or adventitia.

Conclusion The experimental data suggest that the Trevo device is highly effective at achieving immediate reperfusion of occluded arteries without causing any clinically significant disruption of vascular integrity.

The use of mechanical clot retrieval devices in acute ischemic strokes (AIS) results in higher reperfusion rates compared with intravenous and/or intra-arterial thrombolysis. ${ }^{1}{ }^{2}$ However, the currently available mechanical devices still fail to achieve recanalization in as many as $20-40 \%$ of proximal arterial occlusion strokes. ${ }^{3-5}$ Furthermore, significant amounts of procedural time are often required before any meaningful reperfusion can be accomplished, resulting in lower proportions of penumbral tissue salvage. We now report on the experimental evaluation of the safety and efficacy of a novel thrombectomy device designed to achieve immediate flow restoration by quickly removing clot.

\section{METHODS}

Four confirmatory animal studies (three device performance; one histopathology) were performed using the Trevo device (Concentric Medical Inc, Mountain View, California, USA) in swine $(n=2)$ and canine $(n=1)$ models of arterial thromboocclusive disease employing autologous thrombin generated clots of varying consistencies by five different operators, as depicted below.

\section{Device description}

The Trevo device was designed for removal of occlusive thrombus in the setting of AIS in vessels ranging from 1.5 to $3.5 \mathrm{~mm}$ in diameter. The device consists of a $180 \mathrm{~cm}$ proximal 0.018 inch core wire with a $75 \mathrm{~cm}$ tapered transition and a closed cell stent-like shaped section at the distal end (figure 1). The overall length of the device is $44 \mathrm{~mm}$ with a diameter of $4 \mathrm{~mm}$. The distal end has a $4 \mathrm{~mm}$ soft atraumatic radiopaque tip designed to allow safe and accurate deployment as well as facilitate fluoroscopic visualization. The distal $10 \mathrm{~mm}$ of the device is tapered to allow for a smooth transition from the soft floppy radiopaque tip to the higher radial force active area of the device, allowing for placement into more distal and smaller vessels. The proximal $10 \mathrm{~mm}$ of the device is also tapered to allow for easy resheathing. The Trevo device has a hydrophilic coating to reduce friction during use. A shaft marker indicates the proximity of the device relative to the microcatheter tip. The orientation of the stent struts differs from the typical orientation of stents used to treat intracranial aneurysms (neck bridging) or intracranial atherosclerosis. In those devices, the broader portion of the struts is in contact with the blood vessel wall in order to optimize coverage and wall apposition. In the Trevo device, the broader portion of the struts has an endoluminal orientation in order to optimized thrombus incorporation.

In addition, the device was designed to have a more constant radial force across the intended vessel diameters. These properties are expected to result in consistent and efficient clot incorporation and retrievability.

\section{Thrombus model}

The clot sizes ranged from 3 to $4 \mathrm{~mm}$ in diameter and from 10 to $15 \mathrm{~mm}$ in length. Clots of different mechanical stiffness were made by using autologous whole blood incubated with varying levels of bovine thrombin (0-8 NIHU/ml of blood) and 


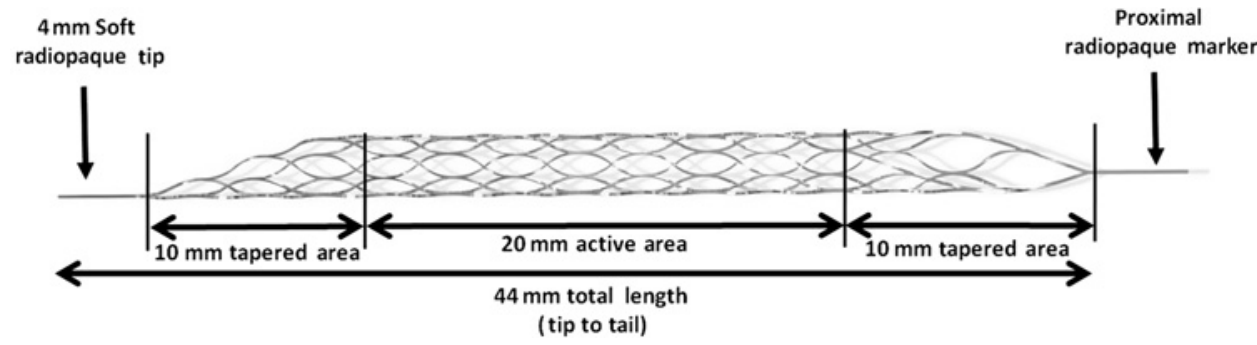

barium (1-2 g/10 $\mathrm{ml}$ of blood) concentrations. A white clot was also prepared using platelet rich plasma isolated from centrifugation of autologous whole blood. The platelet rich plasma was incubated with bovine thrombin ( $8 \mathrm{NIHU} / \mathrm{ml}$ of plasma) and barium ( $1 \mathrm{~g} / 10 \mathrm{ml}$ of plasma). The clot fragments were selectively introduced into the target vasculature, as described below. This radio-opaque thrombus model allowed the authors to evaluate for thrombus fragmentation and/or distal embolization during passing, deploying and retrieval of the device, as previously described by Gralla and colleagues. ${ }^{6}$

Animal models, procedural technique and measures of outcome All procedures were approved by the local Institutional Care and Use Committees and were conducted in accordance with guidelines established by the Animal Welfare Act (http://www. aphis.usda.gov/ac/publications.html). Three Yorkshire swine weighing approximately $30 \mathrm{~kg}$ and one mongrel dog weighing approximately $20 \mathrm{~kg}$ were used. In all cases, anesthesia was maintained with mechanical ventilation of oxygen containing $2 \%$ isoflurane. A heparin bolus $(100 \mathrm{U} / \mathrm{kg})$ was intravenously administered, and anticoagulation was sustained with maintenance administration of $500 \mathrm{U}$ every hour to maintain the activated clotting time above 250 . Femoral artery access was subsequently obtained, and an $8 \mathrm{~F}$ guiding catheter $(6 \mathrm{~F}$ in the canine model) was placed in the target vessel using a 0.035 inch hydrophilic wire.

For the efficacy analysis, 16 thrombi of varying consistencies were injected through a syringe into the guiding catheter and allowed to embolize distally into the swine internal maxillary (IMA, $n=6)$, lingual $(n=3)$ and forelimb $(n=4)$ arteries as well as the canine external carotid $(n=1)$ and vertebral $(n=2)$ arteries. The thrombi were allowed to mature in place for at least $30 \mathrm{~min}$. The diameter of the target vessels ranged between 2 and $3.5 \mathrm{~mm}$, which are comparable with the size of human middle cerebral, basilar and distal intracranial internal carotid arteries. Vessel occlusion was confirmed by digital subtraction angiography and assessment of a Thrombolysis in Myocardial Infarction (TIMI) flow grade of 0 or 1 (persistent occlusion or trickle flow). ${ }^{7}$ A 2.5 F MC18 Plus microcatheter (Concentric Medical Inc) was navigated over a 0.014 microwire across the occlusive thrombus. The wire was removed and a microcatheter contrast injection was performed to confirm an endoluminal position distal to the occlusion. The Trevo device was then advanced into the microcatheter spanning the whole length of the occlusive thrombus. The Trevo device was then deployed by slowly retracting the microcatheter while maintaining constant forward tension on the Trevo pusher wire. The unsheathing of the device radially displaces the thrombus against the blood vessel wall resulting in immediate flow restoration. A guiding catheter angiogram was performed to assess the degree of reperfusion. A thrombus retrieval attempt was then performed within $60 \mathrm{~s}$ of deployment by slowly withdrawing the Trevo device and the microcatheter as a unit into the guiding catheter under fluoroscopic guidance. At that point, aspiration was performed using a $60 \mathrm{ml}$ syringe without any flow arrest (eg, balloon guide catheters were not used). Control angiography was performed after each pass of the device to assess the degree of recanalization according to the TIMI scale (primary efficacy outcome). In addition, the degree of device-clot incorporation was evaluated by macroscopic examination of the device and retrieved thrombi. Finally, a high resolution flat panel threedimensional CT built from 610 projection images acquired over a $200^{\circ}$ arc (Philips Healthcare, Best, The Netherlands) was performed with the device deployed against an occlusive thrombus in the swine lingual artery to further define the in vivo device-thrombus-vessel interaction.

For the safety analysis, the Trevo device was deployed through the MC18 Plus microcatheter into the swine right IMA (diameter $3.0 \mathrm{~mm}$ ), right ascending pharyngeal artery (APA $2.4 \mathrm{~mm}$ ) and left APA (2.2 $\mathrm{mm})$ and then retracted as a system, six times in each vessel. At the conclusion of the procedure, the animal was sacrificed and tissue samples of the treated vessels were collected. The specimens were processed for histopathological analysis including staining with hematoxylin-eosin and an elastin (Movat Pentachrome) stain. To assess the degree of arterial injury, ordinal data were collected for multiple parameters to include the presence of (1) endothelial loss, (2) platelets/ fibrin on the denuded luminal surface, (3) hemorrhage in the media, (4) hemorrhage in the adventitia and (5) medial injury using a semiquantification score system (table 1). Values were calculated for every section and level and reported as an overall mean of each vessel, ranging from 0 (no injury) to 20 (highest possible degree of injury). In another safety evaluation test, the device was deployed in the canine basilar artery to assess for any angiographic evidence of vessel injury in a small $(1.5 \mathrm{~mm}$ diameter) thin walled pial vessel.

\section{RESULTS}

A total of 16 clots of variable hardness and consistencies were implanted in a variety of vascular settings, as described above. TIMI 2-3 reperfusion was achieved in all vessels immediately after device deployment (figure 2A,B). There was no angiographic evidence of dissection, contrast extravasation or thrombus formation, but varying degrees of vasospasm were noted in most of the manipulated swine vessels throughout the procedure. This occurred during guiding catheter, wire and microcatheter manipulation but was more significant after the retrieval of the device. Overall, the degree of vasospasm was much milder in the canine than in the swine model. Partial fragmentation of the radio-opaque clot was noted in some cases after microcatheter crossing and/or device deployment but in all cases the device retrieved the entire thromboembolic material after one $(n=15)$ or two $(n=1)$ passes resulting in a $100 \%$ TIMI/ Thrombolysis in Cerebral Infarction (TICI) 3 flow reperfusion rate (figure $2 \mathrm{C}, \mathrm{D}$ ). In the case that required two device passes, a small fragment of a soft clot was lost at a vessel bifurcation during the initial pass, resulting in incomplete reperfusion (TIMI/TICI 2). This small fragment was easily retrieved during 
Table 1 Semiquantitative analysis of histopathologic changes in vessels treated with the Trevo device

\begin{tabular}{|c|c|c|c|c|c|}
\hline Score & 0 & 1 & 2 & 3 & 4 \\
\hline \multicolumn{6}{|l|}{ Endothelium } \\
\hline Endothelial loss (\%) & None & $<25 \%$ of the circumference & $25-50$ & $51-75$ & $>75$ \\
\hline \multicolumn{6}{|l|}{ Thrombus } \\
\hline Thrombus (fibrin/platelet) & None & Minimal, focal & Mild, multifocal & Moderate, regionally diffuse & $\begin{array}{l}\text { Severe, marked diffuse or tota } \\
\text { luminal occlusion }\end{array}$ \\
\hline \multicolumn{6}{|l|}{ Hemorrhage } \\
\hline Adventitial & None & Focal, occasional & Multifocal and regional & Regionally diffuse & $100 \%$ red blood cells \\
\hline Medial & None & Focal, occasional & Multifocal and regional & Regionally diffuse & $100 \%$ red blood cells \\
\hline \multicolumn{6}{|l|}{ Medial injury } \\
\hline Medial injury (device induced) & None & $\begin{array}{l}\text { Focal disruption } \\
\text { of the IEL }\end{array}$ & $\begin{array}{l}\text { Widespread disruption } \\
\text { of the IEL }\end{array}$ & $\begin{array}{l}\text { Medial tear not extending } \\
\text { beyond the EEL }\end{array}$ & $\begin{array}{l}\text { Medial tear with involvement } \\
\text { of the EEL }\end{array}$ \\
\hline
\end{tabular}

$\mathrm{EEL}$, external elastic lamina; IEL, internal elastic lamina.

the second pass, resulting in TIMI/TICI 3 flow. Otherwise, the thrombus-device interaction during the retrieval process appeared relatively unchanged from the post-deployment status with no obvious additional fragmentation, stretching or compression of the thrombus until it reached the guiding catheter tip. There was some additional fragmentation but no loss of thrombus at the guiding catheter tip during retrieval into the guiding catheter under aspiration. Macroscopic examination of the device and retrieved thrombotic materials revealed that the device incorporated clots of both soft and hard consistencies (figure 3A,B). Clot incorporation by the device was also appreciated in vivo by the high resolution flat panel CT examination (figure 4A,C).

Histopathological analysis was conducted in three swine external carotid branches ranging from 2.2 to $3 \mathrm{~mm}$ in diameter. Five sections with six levels each were analyzed in each of the
Figure 2 Examples of immediate flow restoration $(A, B)$ and thrombus retrieval (C, D). (A) Pretreatment angiography of the swine right lingual artery. Black arrow: point of complete occlusion (Thrombolysis in Myocardial Infarction (TIMI)/Thrombolysis in Cerebral Infarction (TICI) 0 flow). White arrowheads: subtracted radiopaque thrombi. (B) TIMI/TICI 3 flow was achieved immediately after deployment of the Trevo device. Arrows: proximal (black) and distal (white) radiopaque markers of the Trevo device. Black arrowhead: microcatheter tip. White arrowheads: areas of residual stenosis due to the presence of thrombi. (C) Pretreatment angiography of the swine left internal maxillary artery. Arrow: point of complete occlusion (TIMI/TIC 0 flow). Arrowhead: subtracted radiopaque thrombus. (D) TIMI/TICI 3 flow was achieved immediately after retrieval of the Trevo device. Arrow: complete recanalization of the internal maxillary artery with no residual stenosis.
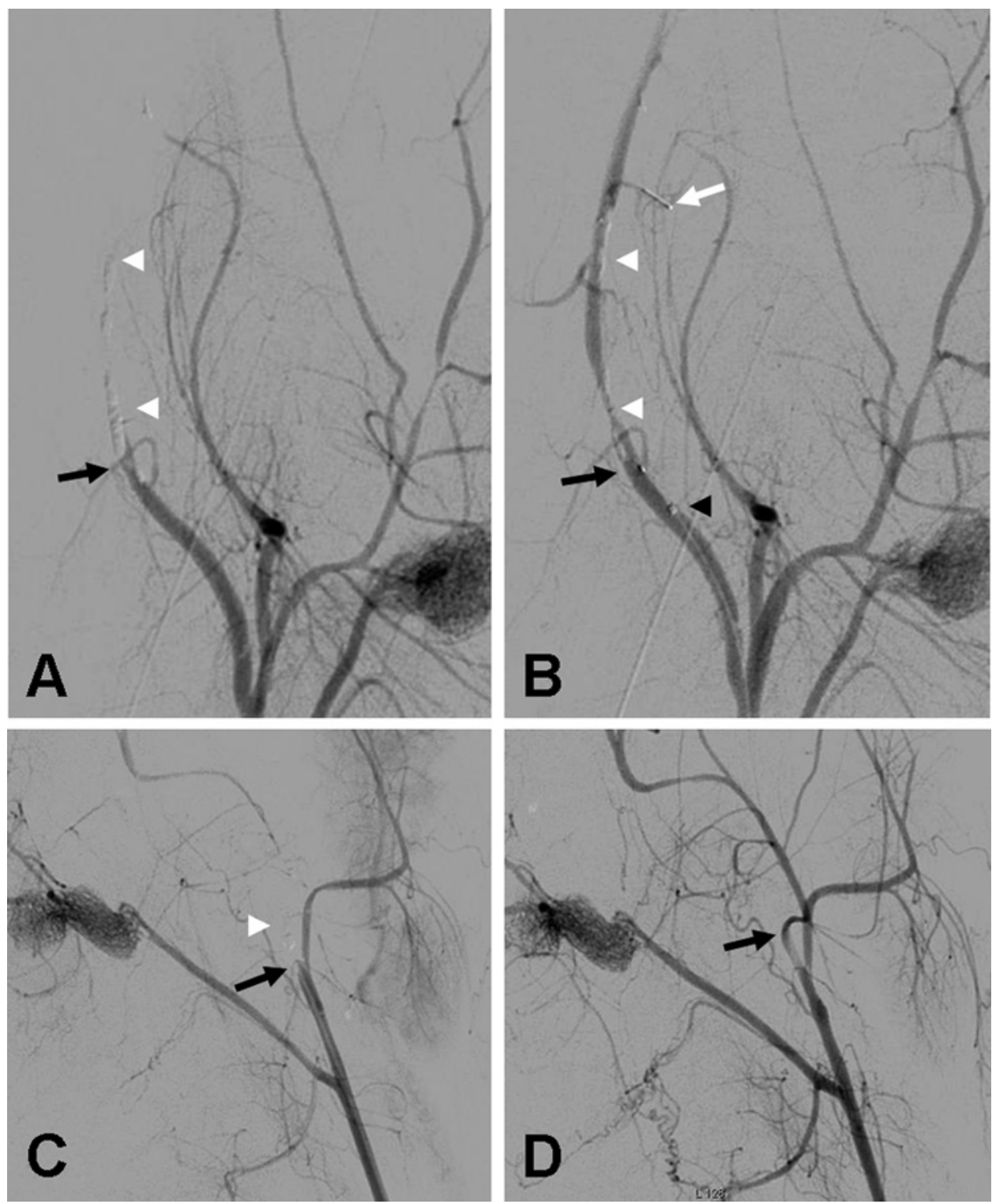


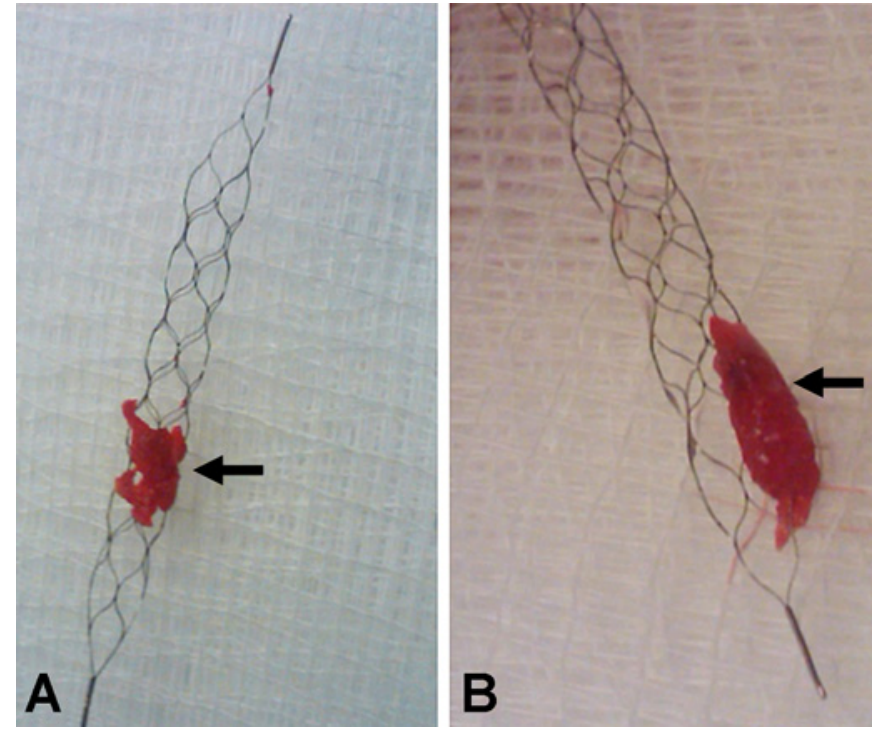

Figure 3 Example of device-clot incorporation with soft $(A)$ and hard (B) thrombi (arrows).

vessels to a total of 90 levels. Essentially, there was severe disruption of the intima but there was no hemorrhage of media/ adventitia or any evidence of extensive arterial injury consisting of transmural medial dissection (tear or perforation) (figure 5). Specifically, the right IMA had a mean injury score of 4.3 out of 20 , due to severe endothelial loss with rare areas of minimal/ focal fibrin/platelet deposition and rare areas of focal disruption of the internal elastic lamina (IEL). The right APA had a meaninjury score of 4.5 out of 20 , due to moderate to severe
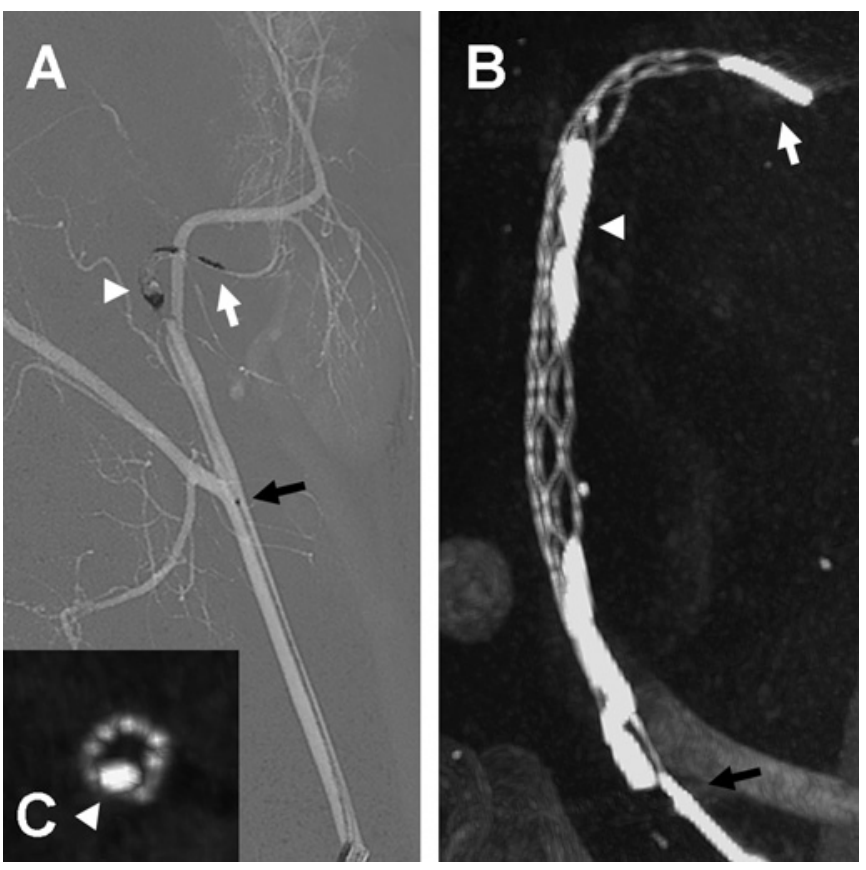

Figure 4 Example of in vivo device-clot-blood vessel interaction. (A) Road map angiography of the swine left lingual artery. (B) High resolution flat panel CT three-dimensional reconstruction. Arrows: proximal (black) and distal (white) radiopaque markers of the Trevo device. Arrowhead: radiopaque thrombus. (C) Axial cut reconstruction of the area identified in (B) by the arrowhead, showing complete incorporation of the radiopaque thrombus (arrowhead) through the struts of the Trevo device.

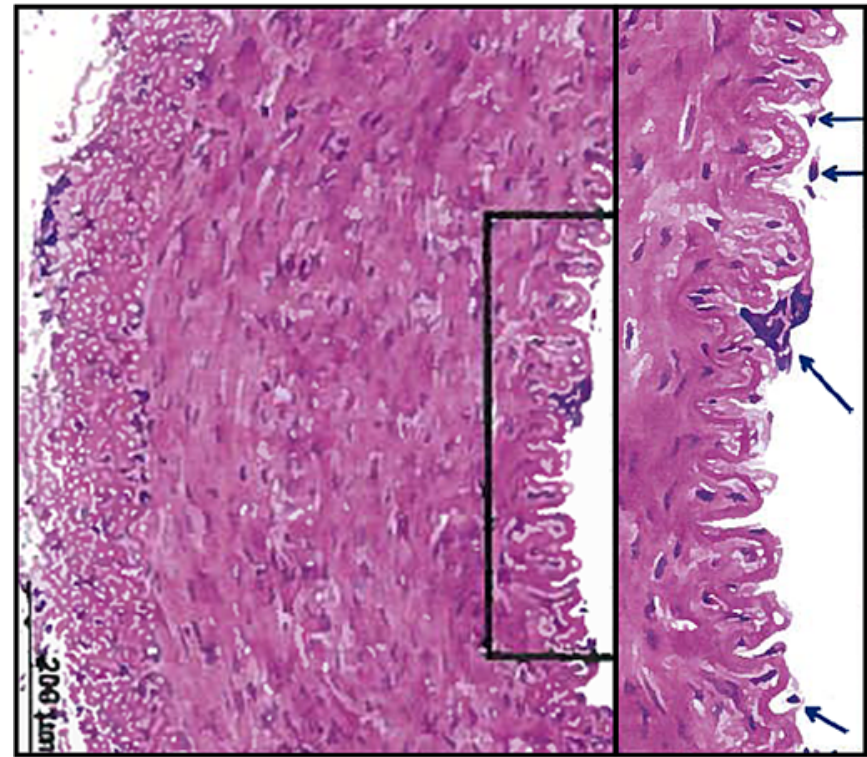

Figure 5 Hematoxylin-eosin staining microphotography of the right ascending pharyngeal artery section showing endothelial cell loss (blue arrows). This was the most common histopathological finding in this study.

endothelial loss with occasional areas of focal or widespread disruption of the IEL. The left APA had a mean injury score of 4.2 out of 20 due to severe endothelial loss with rare areas of minimal focal fibrin/platelet deposition and rare areas of disruption of the IEL.

The Trevo device was navigated into the canine basilar artery without any technical difficulties. As expected from purposefully oversizing the device, its deployment/retrieval resulted in severe self-limiting vasospasm of the basilar artery but there was no angiographic evidence of arterial dissection, perforation, rupture or thrombosis.

\section{DISCUSSION}

We have reported on the experimental data supporting the safety and efficacy of the Trevo device, a novel mechanical device designed to treat AIS patients. The Trevo device belongs to a new class of thrombectomy devices increasingly known as 'stentrievers' due to their resemblance to intracranial stents. This new technology has several theoretical advantages in relation to the currently employed approaches. The use of intracranial stents in AIS has been associated with rapid flow restoration and high recanalization rates. ${ }^{1}$ However, this technique is limited by the need for an aggressive antithrombotic regimen to avoid acute in-stent thrombosis which obviously increases the risk of hemorrhagic complications in the AIS setting. ${ }^{8}$ Another important limitation to the use of intracranial stents in AIS is that, unlike acute coronary syndromes where plaque rupture in an underlying atheroma is the most frequent offender, most cases of acute intracranial arterial occlusions are related to an embolus in the absence of any prior in situ vascular pathology. In this setting, placement of a permanent implant may incur an additional morbidity without any advantage over thrombus removal.

The idea of using stents or stent-like devices as clot retrievers is not novel. The Neuronet device (Guidant Corp, Santa Clara, California, USA) was a microguidewire based laser cut nitinol basket that closely resembled a closed cell stent attached to a shapeable platinum tipped wire. This device, which probably 
constituted the first generation of 'stentrievers', had already shown highly promising results nearly a decade ago. ${ }^{9-11}$ Unfortunately, a planned large clinical trial with the Neuronet device was never completed and the device is no longer available. The idea of using a closed cell stent (eg, Enterprise, Codman \& Shurtkeff Inc, Raynham, MA, USA) for clot or foreign body removal was subsequently tested in vitro and in in vivo models which confirmed the high potential of this approach. ${ }^{12}$ Finally, the Solitaire $A B$ device, initially conceptualized as an aneurysm neck bridging stent, has been used in an off-label manner as a thrombectomy device and demonstrated unparallel speeds of reperfusion and recanalization rates. ${ }^{13}$ This led to minor design changes to produce the Solitaire FR device which is currently CE marked in Europe for the treatment of AIS.

The 'stentriever' concept keeps all of the major advantages of intracranial stenting, including immediate flow restoration and the possibility of using adjuvant intra-arterial pharmacological therapy to dissolve the circumferentially displaced thrombus, but it also overcomes most of the limitations associated with stent use by eliminating the need for postprocedural antithrombotic therapy as well as the risks associated with a long term implantation. In addition, this approach allows for quick and reliable clot removal which at least theoretically should result in lower chances of early vessel re-occlusion compared with pharmacological and other mechanical approaches, such as thrombus disruption, angioplasty and stenting. Our findings are fully consistent with these notions: we have achieved 100\% reperfusion rate without any evidence of early re-occlusion.

Stentrievers also have potential advantages over many of the currently available thrombectomy devices. In contrast with other devices such as the Merci device which have a more fixed diameter, stentrievers gradually expand in diameter as they are withdrawn into the guiding catheter through the more proximal larger intracranial and cervical vessels and as such they oppose the blood vessel throughout the retrieval process. This prevents the retrieved thrombi from dislodging from the device and reembolizing to the cerebrovasculature. Therefore, they may obviate the need for proximal vessel occlusion with a balloon guiding catheter, a step that often adds time and technical challenges to thrombectomy.

Our study carries all of the limitations inherent to the inability of animal models to fully reproduce the challenges encountered in humans. While the diameters of the vessels treated in our study were fairly consistent with intracranial human arteries, neither the canine nor swine model can simulate the same degree of tortuousity encountered in the human cerebrovasculature. Another limitation of the porcine model is their aggressive coagulation system which is not representative of humans. ${ }^{14}$ Consequently, it is useful to provide anticoagulation $^{15}$ or antiplatelet ${ }^{16}$ therapy in this model system during the evaluation of thrombectomy. It is possible that the use of anticoagulation in our study may have prevented re-occlusion in the setting of device related endothelial injury. Even though we tested a wide range of clot consistencies, it is unlikely that we could mimic the broad variety of situations that can occur during human strokes. Moreover, the histologic characteristics of our thrombus model are not necessarily consistent with those of thromboemboli recovered from patients with stroke. However, we have addressed one of the main concerns related to the use of stentrievers: the theoretical risk of causing clinically significant vascular injury by 'dragging a deployed stent' through the cerebrovasculature. We have addressed this issue in the current study by carrying out a detailed histopathological analysis of the treated vessels.
The histological findings were within the limits of similar endovascular procedures with no significant adverse concerns attributed to the Trevo device. In this regard, there is a paucity of literature about the risks of vascular injury after the use of stroke thrombectomy devices. Jahan reported on the comparison of the performance of the Solitaire revascularization device (ev3 Neurovascular Inc, Irvine, California, USA) versus the Merci retrieval system (Concentric Medical, Inc) in restoring blood flow in swine costocervical and ascending pharyngeal arteries. ${ }^{17}$ The Solitaire device established immediate flow restoration following deployment in five of six vessels and retrieved clot resulting in TIMI 3 flow in six of six vessels. The Merci device achieved TIMI 2 flow in two of three vessels and TIMI 3 flow in one of three vessels. Similar to our study, vasospasm was noted for all Solitaire and Merci procedures. There was no angiographic evidence of any vascular injury or thrombus formation with either device. Safety was evaluated by performing three deployments of the Solitaire and Merci devices in the contralateral renal arteries which measured approximately $2 \mathrm{~mm}$. Histological evaluation of the renal arteries showed acute inflammation and hemorrhage in the adventitia of both treated vessels. Both vessels had some degree of internal elastic lamina degeneration, more extensive with the Merci device and only focally with the Solitaire device. There was in situ clot formation with the Merci device. This was not seen on angiography but noted on histology. A similar animal study evaluating the Solitaire device was recently reported by Mordasini et al using a swine model of thromboembolism. These authors reported complete (TICI 3) and partial (TICI $2 b$ ) recanalization rates of $86.7 \%$ and $13.3 \%$, respectively. ${ }^{16}$ Our findings indicate that the safety of the Trevo device is at least comparable with the Solitaire and Merci devices.

In summary, our experimental data suggest that the Trevo device is highly effective at achieving immediate reperfusion of occluded arteries without causing any clinically significant disruption of the vascular integrity.

Acknowledgments The authors would like to acknowledge Dr. Elena Ladich, MD (CV Path Institute, Inc. Gaithersburg, MD) for invaluable work on the histopathological evaluation.

Disclosures Raul G. Nogueira is a member of the Scientific Advisory Boards (SAB) for Concentric Medical Inc., ev3 Neurovascular Inc., CoAxia Inc., and Rapid Medical Inc. Elad I Levy receives research grant support, other research support (devices), and honoraria from Boston Scientific and research support from Micrus Endovascular and ev3; has ownership interests in Intratech Medical Ltd. and Mynx/Access Closure; serves as a consultant on the SAB to Cordis Neurovascular; serves as a consultant per project and/or per hour for Micrus Endovascular, ev3, and TheraSyn Sensors, Inc; and receives fees for carotid stent training from Abbott Vascular and ev3. Dr Levy receives no consulting salary arrangements. All consulting is per project and/or per hour Matthew J. Gounis serves as a consultant for Micrus Endovascular, Codman Neurovascular, and Soteira Inc. He also currently receives research support from Philips Healthcare, Guerbet, and the National Institutes of Health. Adnan H Siddiqui has received a research grant from the University at Buffalo, is a consultant to Codman/Cordis, Concentric Medical, ev3, and Micrus Endovascular, serves on speakers' bureaus for Cordis and Genentech, and has received honoraria from Genentech, Neocure, an American Association of Neurological Surgeons' course, and an Emergency Medicine Conference, and from Cordis for training other neurointerventionists. Gary Duckwiler has stock ownership in Concentric Medical Inc.

Funding Concentric Medical, Inc. funded this project; however, all the performance data analysis and interpretation was performed by the authors, independent of Concentric Medical's input or interpretation.

\section{Competing interests Yes}

Provenance and peer review Not commissioned; externally peer reviewed.

\section{REFERENCES}

1. Nogueira RG, Schwamm LH, Hirsch JA. Endovascular approaches to acute stroke, part 1: drugs, devices, and data. AJNR Am J Neuroradiol 2009;30:649-61. 
2. Nogueira RG, Yoo AJ, Buonanno FS, et al. Endovascular approaches to acute stroke, part 2: a comprehensive review of studies and trials. AJNR Am J Neuroradiol 2009;30:859-75.

3. Smith WS, Sung G, Starkman S, et al. Safety and efficacy of mechanical embolectomy in acute ischemic stroke: results of the MERCI trial. Stroke 2005;36:1432-8.

4. Smith WS, Sung G, Saver J, et al. Mechanical thrombectomy for acute ischemic stroke: final results of the Multi MERCI trial. Stroke 2008;39:1205-12.

5. Penumbra Pivotal Stroke Trial Investigators. The penumbra pivotal stroke trial: safety and effectiveness of a new generation of mechanical devices for clot removal in intracranial large vessel occlusive disease. Stroke 2009;40:2761-8.

6. Gralla J, Schroth G, Remonda L, et al. A dedicated animal model for mechanical thrombectomy in acute stroke. AJNR Am J Neuroradiol 2006;27:1357-61.

7. The Thrombolysis in Myocardial Infarction (TIMI) trial. Phase I findings. TIMI Study Group. N Engl J Med. 1985;312:932-6.

8. Levy El, Mehta R, Gupta R, et al. Self-expanding stents for recanalization of acute cerebrovascular occlusions. AJNR Am J Neuroradiol 2007;28:816-22.

9. Mayer TE, Hamann GF, Brueckmann H. Mechanical extraction of a basilar-artery embolus with the use of flow reversal and a microbasket. $N$ Engl J Med 2002;347:769-70.

10. Nesbit GM, Luh G, Tien R, et al. New and future endovascular treatment strategies for acute ischemic stroke. J Vasc Interv Radiol 2004;15:S103-10.
11. Bergui M, Stura G, Daniele D, et al. Mechanical thrombolysis in ischemic stroke attributable to basilar artery occlusion as first-line treatment. Stroke 2006;37:145-50.

12. Wakhloo AK, Gounis MJ. Retrievable closed cell intracranial stent for foreign body and clot removal. Neurosurgery 2008;62(5 Suppl 2):0NS390-3.

13. Castano C, Dorado L, Guerrero C et al. Mechanical thrombectomy with the Solitaire $A B$ device in large artery occlusions of the anterior circulation: a pilot study. Stroke 2010;41:1836-40.

14. Galvez A, Badimon L, Badimon JJ, et al. Electrical aggregometry in whole blood from human, pig and rabbit. Thromb Haemost 1986;56:128-32.

15. Lang EV, Kulis AM, Villani M, et al. Hemolysis comparison between the OmniSonics OmniWave Endovascular System and the Possis AngioJet in a porcine model. J Vasc Interv Radiol 2008;19:1215-21.

16. Mordasini P, Frabetti N, Gralla J, et al. In vivo evaluation of the first dedicated combined flow-restoration and mechanical thrombectomy device in a swine model of acute vessel occlusion. AJNR Am J Neuroradiol 2011;32:294-300.

17. Jahan R. A novel, self expanding, fully retractable mechanical thromboembolectomy device for treatment of acute ischemic stroke. Paper Presented at: 5th Annual Meeting of the Society of Neurolnterventional Surgery (SNIS). Lake Tahoe, CA 2008.

\section{Have confidence in your decision making.}
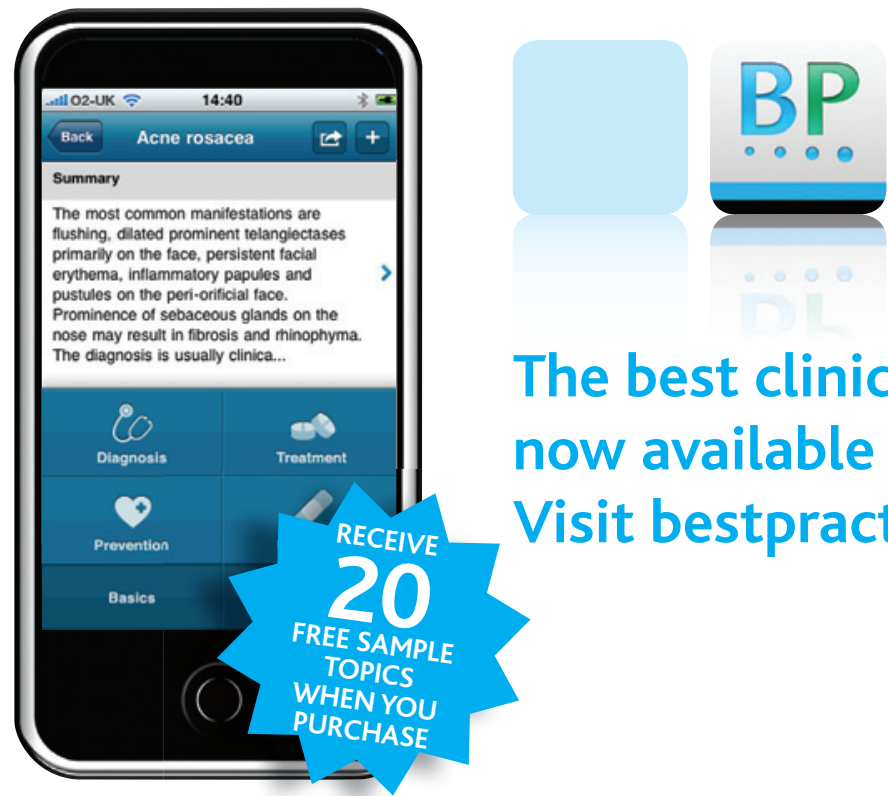

The best clinical decision support tool is now available as an app for your iPhone. Visit bestpractice.bmj.com/app 